\title{
Interactive Multimedia System Using Serious Game for Users with Motor Disabilities
}

\author{
Antoni Jaume-i-Capó and Andreja Samčović
}

\begin{abstract}
User demotivation is habitual in a long-term rehabilitation process, because this process usually consists in repetitive and intensive activities which become boring after hundreds of sessions. Research studies have shown that serious games help to motivate users in a rehabilitation process. Motor rehabilitation consists of body movements that can be captured and patients can have difficulties in holding physical devices. For this reason, therapy studies include vision input devices and propose what features are desirable for rehabilitation serious games. In this paper, we present a case of success, an interactive multimedia system using a serious game to improve abilities of patients with cerebral palsy.
\end{abstract}

Keywords - health care, kinect, motor rehabilitation, multimedia, serious game, vision-based interaction.

\section{INTRODUCTION}

$\mathrm{I}$ $\mathrm{N}$ A long-term rehabilitation process, user demotivation is habitual, because it usually consists in repetitive and intensive activities which become boring after hundreds of sessions. As a consequence, a user may not concentrate on a therapy program, so therapy loses effectiveness, because rehabilitation results are better when users are motivated [1]-[2]. Besides, when we focus on rehabilitation for maintaining users' capacities, where users rarely increase their capacities, demotivation can cause patient's resignation [3]. Moreover, if a user also has a cognitive disability, therapy is more difficult to be continued by the user.

Research studies have shown that serious games help to motivate users in a rehabilitation process [4] defining a

Paper received August 18, 2015; revised October 1, 2015; accepted October 7, 2015. Date of publication November 15, 2015. The associate editor coordinating the review of this manuscript and approving it for publication was Prof. Irini Reljin.

This paper is a revised and expanded version of the paper presented at the 22th Telecommunications Forum TELFOR 2014.

This work was partially supported by the projects 28/2011 (Ajudesgrupscompetitius UGIVIA) granted by the Govern de les Illes Balears; TIN2012-35427 of the Spanish Government, with FEDER support; EUROWEB Project (http://www.mrtc.mdh.se/euroweb) funded by the Erasmus Mundus Action II programme of the European Commission, and the Ministry of Science, Education and Technological Development of the Republic of Serbia (projects TR 32025 and 32048). We also thank the Mathematics and Computer Science Department at the University of the Balearic Islands for its support.

Antoni Jaume-i-Capo is with the Department de Ciencies Matematiques i Informatica, University de les Illes Balears, Palma, Spain (phone: +34 971 259721; e-mail: antoni.jaume@uib.es).

Andreja Samčović is with the Faculty of Transport and Traffic Engineering, University of Belgrade, Serbia (phone: +381 11 3091217; email: andrej@sf.bg.ac.rs). serious game as a video game that allows the player to achieve a specific purpose using the entertainment and engagement component provided by the experience of the game. The cognitive and motor activities required by video games engage a user's attention [5]. In addition, a user focuses his/her attention on the game and this helps to forget that he is making therapy [6]-[7].

Therapy studies have included the motion-based input devices for serious games, such as EyeToy (Sony Computer Entertainment, UK), Kinect (Microsoft, USA) and Wiimote (Nintendo, Japan). They concluded that users couldn't use existing commercial video games for these devices during rehabilitation therapy, because they were designed for users with full capacities [8]-[11]. Moreover, in [8] are numerated the problems of commercial video games when used in rehabilitation: mainly target upperbody gross motor function, lack the support for task customizations, grading, and quantitative measurements. In order to solve these problems, researchers began to develop their own games.

In previous work, we can find a wide range of serious games for different types of rehabilitation. Video games for balance rehabilitation were presented where therapy was performed by a center-of-pressure [12], and in [13] where a game was designed for older adults while incorporating appropriate balance exercises. Regarding upper limb rehabilitation, in [14] was presented a video game based movement therapy which aims to encourage stroke patients with upper limb motor disorders to practice physical exercises. Reference [15] shows a virtual reality (VR) system for stroke patients, in [3] were designed several serious games which used low-cost webcams as input technology to capture data of user's movements, whereas in [16] a simple game was created in which the patient tried to move a colored circle from an initial position to a goal position using a robotic device designed for arm rehabilitation.

Reference [17] implements a haptic glove serious game for finger flexion and extension therapy, in [18] were presented several home-based serious games which used a webcam and a Wiimote, and in [8] was designed a lowcost VR-based system using Wiimote. In [19] was presented a vision-based interactive system to improve the balance and postural control of adults with cerebral palsy, which we will detail in next sections.

Recent research studies proposed what features were desirable for rehabilitation serious games. In [20] are proposed target audience, visibility and feedback as important human factors; in [3] were identified two principles of game design theory which have particular relevance for rehabilitation: meaningful play, the relationship between player's interactions and system 
reaction, while challenge, maintaining an optimum difficulty, was important in order to engage the player. In [4] were identified, based on the literature reviewed, the following important main criteria for the classification of serious games in the rehabilitation area: application area, interaction technology, game interface, number of players, game genre, adaptability, performance feedback, progress monitoring, as well as game portability. In [18], it was concluded that serious games must ensure that patients were correctly performing and must provide a motivating context for therapy, in order to have maximum impact on the rehabilitation process. Reference [21] presents implementations guidelines for developing vision-based motor-rehabilitation serious games, based on related work and their experience, in order to help other researchers in this field. They stated that the success of this type of serious games depended on seven design issues: development paradigm, interaction mechanism, interactive elements, feedback, adaptability, monitoring, and clinical evaluation.

In this paper we show first the advanced multimedia technologies used for serious games. The remainder of this paper is organized as follows. Section III exploits the benefits of using vision-based tacking as input for interactive multimedia systems and we present also these types of systems that we used in the rehabilitation process. Section IV presents a case of success, a vision-based interactive system for balance and postural control of adults with cerebral palsy. The experimental procedure is described in the following part. Finally, the last section presents some conclusions.

\section{MultimediA TECHNOLOGIES FOR SERIOUS GAMES}

The latest technologies mean that synthetic characters and emergent narrative can be developed to create improvised educational scenarios:

- 3D (three-dimensional) animated video games;

- Virtual reality;

- Augmented reality;

- Mixed reality.

The serious games application is intended to help professionals, as well as enable users to enjoy themselves through a straightforward, real interaction while learning how to cope in several real social situations.

Main technologies used in serious games development are: augmented reality and virtual reality.

Augmented reality is currently being used in such areas as architecture, where it is employed to show the final result of a project still in its planning phase or the virtual reconstruction of a building in ruins. In the educational field it has been used more frequently for applications in museums or theme parks. One of augmented reality's main attractions is that any virtual figure modeled in 3D can be incorporated into one or more real patterns. In addition, any move or turn of this pattern is the equivalent of moving or turning the virtual figure. Thanks to augmented reality, serious games scenarios combine the real environment scenario with virtual objects, displaying a single mixed environment to the user, thus making him feel more involved and identified.

Virtual reality technology is used for the development of serious games. Using this technology, a wide variety of areas, such as medicine or education, has been developed from serious games. Thanks to the use of this technology it has been possible to reconstruct different situations or environments that would be impossible to recreate in the real world, such as a traumatic situation, and thus help in the rehabilitation of individuals [22].

Through virtual reality, it can be managed to get the user to be involved completely; this is achieved by using different hardware that lets the user to interact with the serious games having this technology implemented. The following are the major types of hardware currently available:

- 3D vision goggles;

-3D LCD (Liquid Cristal Display) screens;

- Data acquisition glove;

- The mouse or keyboard;

- Sensor body;

- Interaction;

- Multiplatform possibilities;

- Mobile devices;

- Consoles;

- Microsoft surface.

There is currently a trend towards creating computerized versions of famous traditional games which can also be played online. The aim is to enrich the user's experience and to make the games an appealing alternative, capable of competing with present-day video games.

Depending on the activities and skills to be worked on with each of the games and the target group, interaction may be one of the aspects that needs to be developed with greatest care. The most appropriate tools are not always the traditional keyboard and mouse. Technology has moved on and there are a host of possibilities opening up in this area. In the future these are likely to be useful for individuals with much reduced mobility.

The latest advances in hardware and software mean that the presentation of serious games has been changed. Video consoles and the serious games that have been developed for them now make it possible to go far beyond the field of pure leisure.

Mobile devices have entered into many aspects of our lives. Their multiple possibilities make them very suitable for education in health and good habits.

Various companies, such as Nintendo and Microsoft, have developed adapted versions and video games of their Wii and Xbox consoles to address aspects such as physical exercise and training in social skills.

Up to recently, video games have predominantly been aimed at entertainment. This trend has been evolving and changing and, in 2002, a group of learning specialists and video game industries were combined/joined forces to face a new challenge in the field of education. As a result, the "Serious Games Foundation" was created, which promotes serious games as a solution for education and health environments. Since then, serious games have played an important role in people's lives. As the number of these games increases, so do the benefits they bring. 
As shown in Fig. 1, thanks to the increased demand of these games, competition between companies is increasing. Therefore, they are forced to research continuously and, as a result, the technology which is used for these games develops at a faster pace.

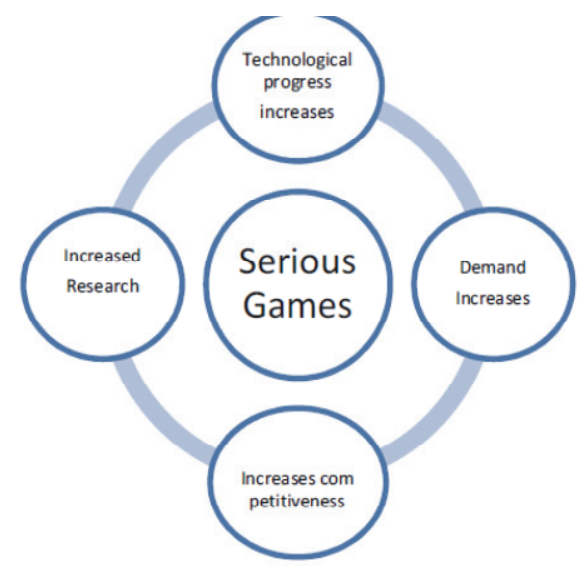

Fig. 1. Serious games demand circle [23].

\section{INTERACTIVE MULTIMEDIA SYSTEM USING SERIOUS GAME}

Our objective was to explore how mirror feedback through interaction could be included into game design in order to observe whether it was possible to improve results in rehabilitation sessions. We performed a user study testing using a game previously designed for balance rehabilitation of cerebral palsy (CP) users to explore mirror feedback in vision-based video games. Participants diagnosed with cerebral palsy with mild to moderate cognitive impairment performed our user study. CP is the most common cause of disabling conditions in children due to the increased survival of low birth-weight infants [24].

The population of adults with $\mathrm{CP}$ is growing, as a result of increased longevity, inspiring new research to improve available therapies to achieve better functional abilities. We chose adults with CP for this reason and because many daily activities require both hands and tasks that typically require bimanual coordination [25]-[26]. The aim was to experimentally test that the feedback of current games was not enough for understanding the game play by users with cognitive impairment and to demonstrate the feasibility and benefit of including mirror feedback in rehabilitation systems.

Visual information from the performance of patient actions is the preferred capture method for two reasons: first, because motor rehabilitation consists of body movements that can be recorded; and second, because vision capture technology is non-invasive and can be used for patients who have difficulties in holding physical devices.

In the usual proposed game environment configuration users stand in front of the screen and interact with the video game using their movements, which is shown in Fig. 2. With this configuration, users can see the serious game while interacting with the game.

Some existing vision-based rehabilitation systems allow the patients to see themselves on a screen, mirror feedback, due to the users standing in front of a screen and interacting with the system using their movements [3], [6],
[10], as can be seen in Fig. 3. Recent research observed that the higher cognitive impairment a user had, the more important feedback was in order to perform correctly the therapy [27]. Results confirmed that in case of disabilities the mirror feedback mechanisms facilitated the interaction in the vision-based systems for rehabilitation.

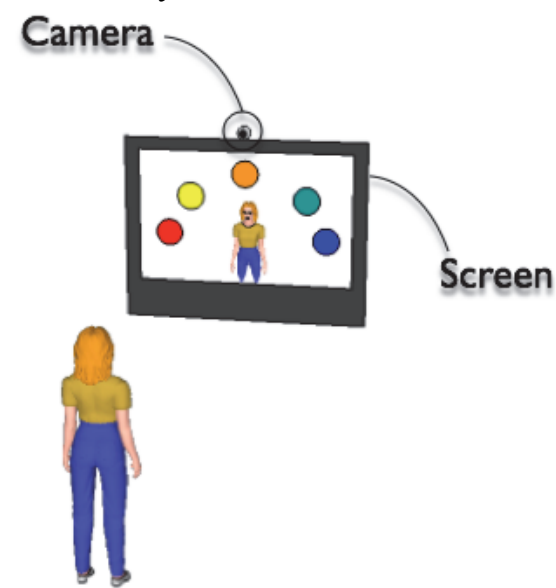

Fig. 2. Game environment configuration.

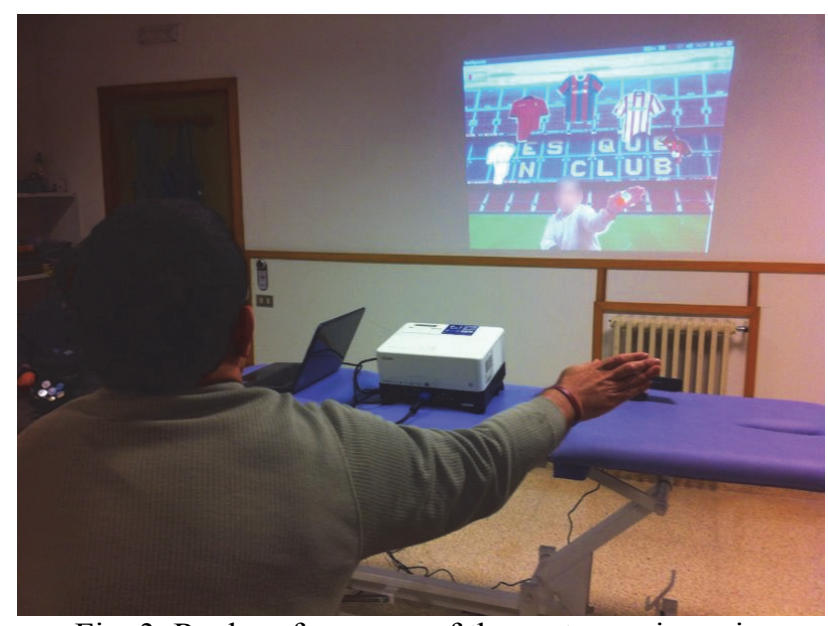

Fig. 3. Real performance of the system using mirror feedback.

\section{EXPERIMENTAL SYSTEM}

The experimental system was designed to improve the balance and postural control of adults with cerebral palsy [8]. The system was based on a video game for balance rehabilitation therapy, designed using the prototype development paradigm and features for rehabilitation with video games [3], [21], [27]: feedback, adaptability, motivational elements and monitoring. Findings demonstrated a significant increase in balance postural control scores resulting in indicators of greater independence for participating adults. Scores improved from 16 to 21 points in a scale of 28 , according to the Tinetti Scale for risk of falls, moving from High Fall Risk to Moderate Fall Risk.

We used an active control therapy [28] as the interaction method for a video game to improve balance and postural control, increase motivation and achieve higher adherence to this long-term therapy. The users must interact with objects that cannot be reached without moving the center of mass beyond the base of support (see Fig. 4). More specifically, users must remove individual items that appear on the screen by reaching each item with one hand. 


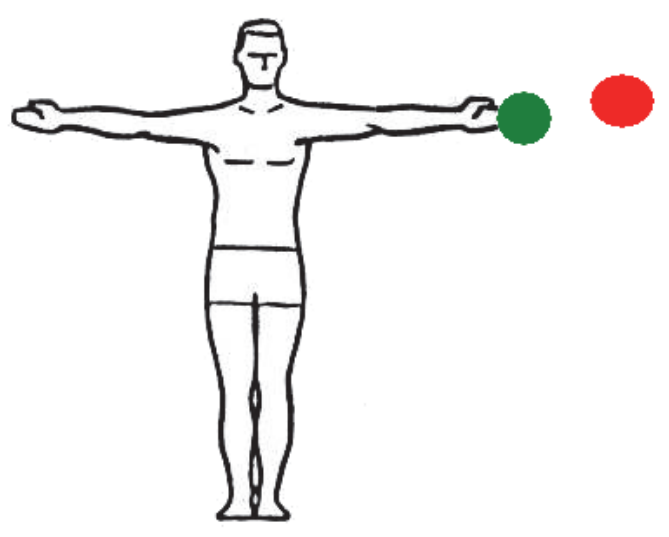

Fig. 4. Users must interact with objects that cannot be reached without moving the center of mass.

The video game responds to the actions of the user through different types of feedback, in order for users to be aware of their current state, as we can see in Fig. 5:

- A pointer is projected on the user's hand, and the part of the interaction object that intersects with the pointer is erased;

- An auditory feedback is played when an interaction object is completely deleted from the screen;

- Mirror feedback, in such a way that the user can see himself/herself on the screen all the time, so the player's position relative to interaction objects is always known.

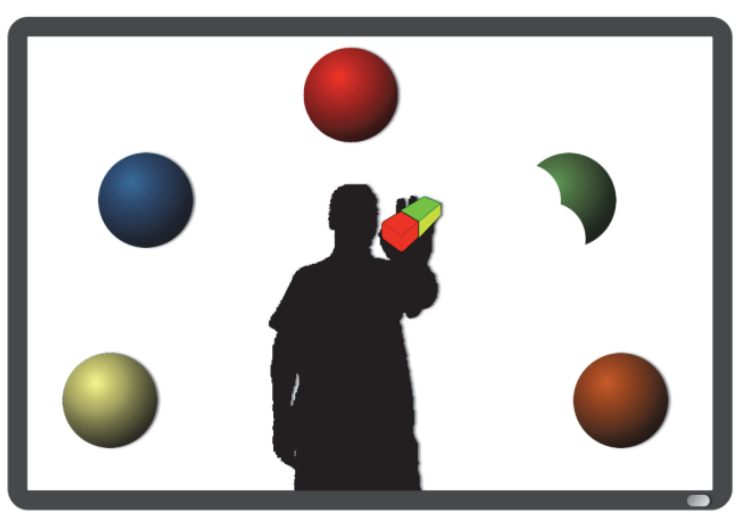

Fig. 5. Representation of types of feedback.

Moreover, when the game ends, the user receives different types of visual and auditory feedback, depending on the end game conditions.

One of the advantages of the presented interactive multimedia system is that it is not necessary to take any measurements. With an adequate combination of the computer vision and virtual view, the user feels that his/her movements in the real world cause actions in the virtual view. The patients are able to understand how their movements perform game actions during the rehabilitation session, thus facilitating the game's correct performance to achieve the rehabilitation goals.

The experimental system was developed using $\mathrm{C}++$ programming language, OpenNI as Kinect device library, $O p e n C V$ as a computer vision library, and $Q t$ as the graphical user interface library.

\section{EXPERIMENTAL PROCEDURE}

In order to explore the importance of mirror feedback in vision-based motor rehabilitation interactive systems, we conducted a user study testing. Specifically, the users tested their own visual representation such as interaction feedback of the video game for rehabilitation.

We were interested in different users' performances in the game interaction enabling or not the explained mirror feedback mechanism; that is, the no-mirror feedback condition was characterized by the absence of such visual feedback.

We used a within-subjects design with the two previously defined feedback conditions: mirror feedback (MF, including the user's own visual representation); nomirror feedback (NF, absence of such visual feedback). In Fig. 6 it is possible to observe the feedback with a mirror, while Fig. 7 represents the interaction without a mirror feedback. The user study was divided into two experiments with two different groups of users: control group (users without disabilities) and users with disabilities.

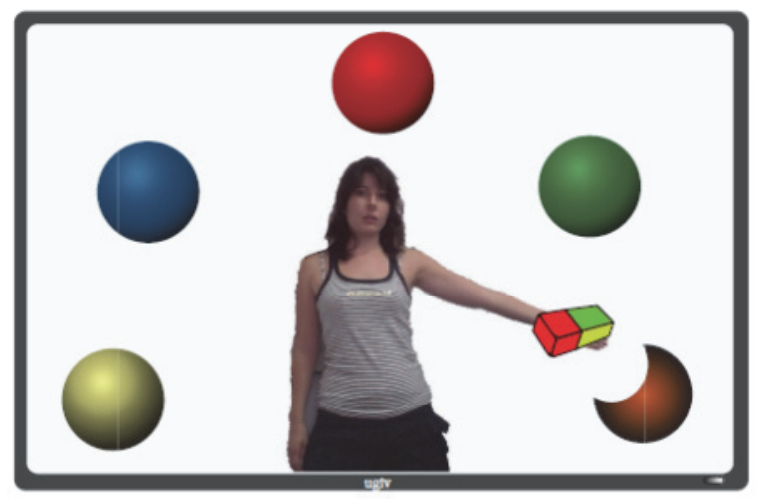

Fig. 6. Experimental feedback with a mirror.

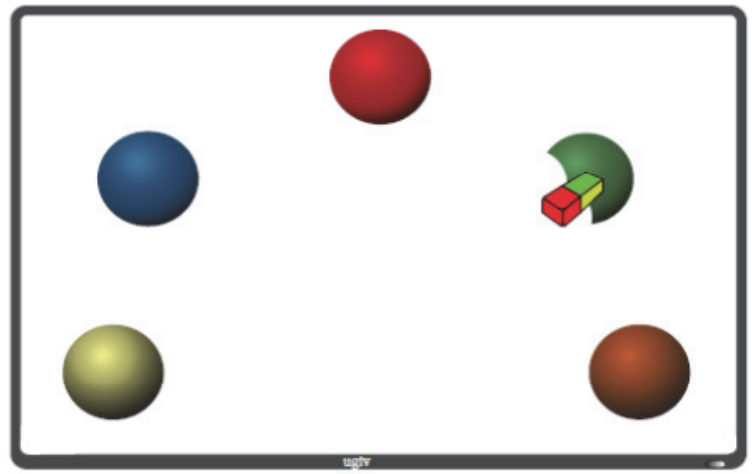

Fig. 7. Experimental feedback without mirror.

The final study population included 8 adults ( 7 males), aged 22 to 41 (mean $(M)=33$ ). A control group was composed of 32 nonpaid volunteers (14 females) aged 19 to $25(M=20.4)$, with no disabilities.

For the control group, the user study started with a brief introduction and a demonstration, together with a demographic questionnaire asking about age and previous use of vision-based interaction applications. Participants played two sessions of the designed computer game with the same conditions that the user with disabilities had, that is, only moving the upper body part to delete the virtual objects with their hands. For each session the order of 
conditions (mirror feedback versus no-mirror feedback) was randomly selected so as to balance both interaction conditions across participants.

Quantitative measures included logged time-tocomplete. The time-to-complete measured the time that users needed to complete the deletion of all virtual objects. In the experiment with the group of users with disabilities, the virtual objects were properly located in order to ensure that all the performances achieved the complete deletion goal. Furthermore, in a final questionnaire, the participants selected their preferred interaction feedback for playing the game. Time-to-complete is related to the efficiency of interaction task. Then, it also has a direct correlation with task completion and the quality of solution.

For users with disabilities, the game was previously tested on a pilot scheme for a two-month period, attending the rehabilitation centre once a week. They practiced the game for at least 20 minutes only with no-mirror feedback condition, and the number of repetitions varied according to participants' tolerance and the physiotherapist's prescription to better manage fatigue. These two months of training were important to ensure a correct understanding of the game and to learn how to carry it out, as well as ensuring a correct parameter adaptation to each user. Once the users correctly understood the game play, participants played two sessions of the designed computer game applying the same procedure as for the control group. Fig. 3 shows real performance of the system in the rehabilitation room using mirror feedback.

Results confirmed our hypothesis that in case of disabilities the mirror feedback mechanisms facilitated the interaction in vision-based systems for rehabilitation. Mirror feedback had better results on the measured times for users with disabilities. They demonstrated that the implementation of mirror feedback by giving patients the possibility of seeing themselves on screen means that they were conscious all the time of the actions performed relative to the video game. In the user study presented, we proved this claim by means of experiments, showing that a significant improvement of users with disabilities results in the game play. We also observed that users with moderate cognitive impairment had higher differences between feedback conditions than users with mild cognitive impairment.

Finally, we want to discuss the relationship between the mirror feedback and the game feel definition used for game design [29]. Game feel is the sensation of the system's response to the player: the kinesthesic qualities of the experience created by coupling with the input device and seeing what happens in the game as a result. In our experience, the input device is the user's own body; therefore, it makes sense that feedback should be related to it as well. In this sense, it is interesting to point out that the results obtained for the control group users (users without disabilities) could be interpreted to mean the mirror feedback mechanism is not significant. However, it should be taken into account that the video game was specifically designed for users with disabilities and its game play was too easy for users without disabilities. It may be interesting to perform another user study with more complex games based on vision-based interaction, in order to properly explore if the introduction of mirror feedback can improve the user experience of vision-based interaction.

\section{CONCLUSIONS}

Serious games are being incoroprated gradually into numerous applications such as health application. Serious games in health have impact on social benefits, namely the therapy concept is changed to a game concept in order to improve the user's motivation. The medical benefits can also be seen because serious games collect some parameters associated with the terapy.

Several studies have demonstrated that serious games help to motivate users in a rehabilitation process, and more recent studies show the benefits of using computer vision as an input mechanism: because motor rehabilitation consists of body movements that can be recorded; and because vision capture technology is non-invasive and can be used for patients who have difficulties in holding physical devices. Also, it is recommended that patients see themselves on a screen (mirror feedback), because in vision-based systems the users stand in front of a screen and interact with the system using their movements.

\section{REFERENCES}

[1] MacLean, N., Pound, P., Wolfe, C., \& Rudd, A. (2002). The concept of patient motivation a qualitative analysis of stroke professionals' attitudes. Stroke, 33(2), 444-448.

[2] Samčović, A., \& Jaume-i-Capo, A. (2013, November). Serious games in health care based on multimedia technologies. In Telecommunications Forum (TELFOR), 2013 21st (pp. 757-760). IEEE.

[3] Burke, J. W., McNeill, M. D. J., Charles, D. K., Morrow, P. J., Crosbie, J. H., \& McDonough, S. M. (2009). Optimising engagement for stroke rehabilitation using serious games. The Visual Computer, 25(12), 1085-1099.

[4] Rego, P., Moreira, P. M., \& Reis, L. P. (2010, June). Serious games for rehabilitation: A survey and a classification towards a taxonomy. In Information Systems and Technologies (CISTI), 2010 5th Iberian Conference on (pp. 1-6). IEEE.

[5] Norman, D. A. (1998). The invisible computer: why good products can fail, the personal computer is so complex, and information appliances are the solution. MIT press.

[6] Flores, E., Tobon, G., Cavallaro, E., Cavallaro, F. I., Perry, J. C., \& Keller, T. (2008, December). Improving patient motivation in game development for motor deficit rehabilitation. In Proceedings of the 2008 International Conference on Advances in Computer Entertainment Technology (pp. 381-384). ACM.

[7] Krichevets, A. N., Sirotkina, E. B., Yevsevicheva, I. V., \& Zeldin, L. M. (1995). Computer games as a means of movement rehabilitation. Disability \& Rehabilitation, 17(2), 100-105.

[8] Anderson, F., Annett, M., \& Bischof, W. F. (2010). Lean on Wii: physical rehabilitation with virtual reality Wii peripherals. Studies Health Technology Information, 154, 229-234.

[9] Deutsch, J. E., et al. (2008). Use of a low-cost, commercially available gaming console (Wii) for rehabilitation of an adolescent with cerebral palsy. Physical therapy, 88(10), 1196-1207.

[10] Flynn, S., Palma, P., \& Bender, A. (2007). Feasibility of using the Sony PlayStation 2 gaming platform for an individual poststroke: a case report. Journal of neurological physical therapy, 31(4), 180189.

[11] Rand, D., Kizony, R., \& Weiss, P. L. (2004, September). Virtual reality rehabilitation for all: Vivid GX versus Sony PlayStation II EyeToy. In 5th Intl. Conf. On Disability, Virtual Environments and Assoc. Technologies (pp. 87-94).

[12] Betker, A. L., Desai, A., Nett, C., Kapadia, N., \& Szturm, T. (2007). Game-based exercises for dynamic short-sitting balance rehabilitation of people with chronic spinal cord and traumatic brain injuries. Physical therapy, 87(10), 1389-1398.

[13] Ryan, R. M., \& Deci, E. L. (2000). Intrinsic and extrinsic motivations: Classic definitions and new directions. Contemporary educational psychology, 25(1), 54-67.

[14] Ma, M., Bechkoum, K. (2008, October). Serious game for movement therapy after stroke. IEEE International Conference on Systems, Man and Cybernetics SMC 2008, 1872-1877. 
[15] Cameirão, M. S., Bermúdez, I. B. S., Duarte Oller, E., \&Verschure, P. F. (2009). The rehabilitation gaming system: a review. Studies Health Technology Information, 145, 65-83.

[16] Colombo, R., Pisano, F., Mazzone, A., Delconte, C., Micera, S., Carrozza, M. C., Dario, P., \& Minuco, G. (2007). Design strategies to improve patient motivation during robot-aided rehabilitation. Journal of Neuro-engineering PMCID, published online.

[17] Jack, D., Boian, R., Merians, A. S., Tremaine, M., Burdea, G. C., Adamovich, S. V. \& Poizner, H. (2001). Virtual reality-enhanced stroke rehabilitation. Neural Systems and Rehabilitation Engineering, IEEE Transactions on, 9(3), 308-318.

[18] Alankus, G., Lazar, A., May, M., \& Kelleher, C. (2010, April) Towards customizable games for stroke rehabilitation. In Proceedings of the SIGCHI Conference on Human Factors in Computing Systems (pp. 2113-2122). ACM.

[19] Jaume-i-Capo, A., Martinez-Bueso, P., Moya-Alcover, B., Varona, J. (2014). Interactive Rehabilitation System for Improvement of Balance Therapies in People with Cerebral Palsy. Neural Systems and Rehabilitation Engineering, IEEE Transactions on, 22(2), 419427.

[20] Jung, Y., Yeh, S. C., \& Stewart, J. (2006, April). Tailoring virtual reality technology for stroke rehabilitation: a human factors design. In CHI'06 extended abstracts on Human factors in computing systems (pp. 929-934). ACM.

[21] Jaume-i-Capó, A., Moyà-Alcover, B., \& Varona, J. (2014). Design Issues for Vision-Based Motor-Rehabilitation Serious Games. In Technologies of Inclusive Well-Being (pp. 13-24). Springer Berlin Heidelberg.
[22] Chen, S., Michael, D. (2005). Serious games: games that educate, train and inform. Thomson Course Technology, USA.

[23] Bartolome, N. A., Zorrilla, A. M., Zapirain, B. G. (2011). Can game-based therapies be trusted? A systematic review of the serious game for health and education. In $16^{\text {th }}$ International conference on Computer games CGAMES 2011, pp. 275-282.

[24] Krigger, K. W. (2006). Cerebral palsy: an overview. The American Family Physician, 73(1), 91-100.

[25] Charles, J., Gordon, A. M. (2006). Development of hand-arm bimanual intensive training (HABIT) for improving bimanual coordination in children with hemiplegic cerebral palsy. Developmental Medicine and Child Neurology, 48(11), 931-936.

[26] Gordon, A. M., Hung, Y. C., Brandao, M., et al. (2011). Bimanua coordination in children with hemiplegic cerebral palsy. A randomized trial. Neurorehabilitation and Neural Repair, 25(8), 692-702.

[27] Jaume-i-Capó, A., Martínez-Bueso, P., Moya-Alcover, B., \& Varona, J. (2014). Improving vision-based motor rehabilitation interactive systems for users with disabilities using mirror feedback. (2014). The Scientific World Journal, Volume 2014, Article ID 964576, 9 pages.

[28] King, L. A., Horak, F. B. (2009). Delaying mobility disability in people with parkinson disease using a sensorimotor agility exercise program. Physical Therapy, 89(4), 384-393.

[29] Swink, S. (2008). Game Feel: A Game Designer's Guide to Virtual Sensation, Morgan Kaufmann. 